\title{
Effects of a formalized collaboration between plastic and orthopedic surgeons in severe extremity trauma patients; a retrospective study
}

\author{
Pehr Sommar ${ }^{1 *}$, Yamin Granberg ${ }^{2}$, Martin Halle ${ }^{1}$, Ann-Charlott Docherty Skogh ${ }^{1}$, Kalle T Lundgren
} and Karl-Åke Jansson ${ }^{3}$

\begin{abstract}
Background: Severe trauma to the extremities often includes a combination of fractures and soft tissue injuries. Several publications support that the patient outcome is better when skeletal stabilization is followed by early soft-tissue coverage. In an effort to optimize the treatment of these patients, we established a formalized collaboration in 2008 between the Departments of reconstructive plastic surgery and orthopedics at the Karolinska University Hospital.

Methods: A retrospective review was conducted for all patients who had suffered severe extremity trauma and received either a free or a pedicled flap for extremity reconstruction. We compared the management of patients $0-4$ years before and $0-4$ years after the collaboration started especially with respect to; choice of flap, time to flap coverage, number of operations/revisions, total in-hospital stay.

Results: After initiation of the collaboration, the number of flaps increased from 13 flaps ( 5 free and 8 pedicled) to 44 flaps (21 free and 23 pedicled). Fewer postoperative revisions was seen, as well as shorter in-hospital stay.

Conclusions: The present study highlights the importance of formalized collaboration between orthopedic and plastic surgeons in severe extremity trauma patients. The concept of an interdisciplinary approach has led to an increased number of trauma patients referred for plastic surgical consultation, an increased number of flaps, fewer postoperative revisions and shorter hospital stay.
\end{abstract}

Keywords: Extremity trauma, Flaps, Orthoplastic collaboration

\section{Introduction}

Severe extremity trauma is often associated with a combination of fractures and soft tissue injuries. It is generally accepted that the outcome is better when skeletal stabilization is followed by early soft-tissue coverage [1-4]. Soft tissue flaps provide the protection and vascularization needed to resist infection and promote bone healing. Meling et al. reported the incidence of all open long bone fractures to be $13 / 10^{5} /$ year 2004-2007 [5]. Weiss et al. found a decreasing incidence of open tibial shaft fractures in the Swedish population, which was $2.3 / 10^{5} /$ year, $1998-2004$ [6]. Because of the complexity of these fractures and the low

\footnotetext{
* Correspondence: pehr.sommar@karolinska.se

'Department of Molecular Medicine and Surgery, Section of Plastic Surgery, Karolinska Institutet and Karolinska University Hospital Stockholm, Stockholm, Sweden

Full list of author information is available at the end of the article
}

incidence rate, an orthoplastic approach has been suggested; i.e. a multidisciplinary collaboration involving both orthopedic and plastic surgery teams $[7,8]$.

Naique et al. demonstrated that severe open tibia fractures treated at dedicated trauma units with both orthopedic and plastic surgery services had lower complication rates and less need for revision surgery compared with those treated initially at hospitals without such combined services [8].

To ensure an orthoplastic approach, we formalized a collaboration between the Departments of reconstructive plastic surgery and orthopedics in April 2008 (Multidisciplinary collaboration MDC). The collaborative protocol agreed upon early attendance of plastic surgeons in extremity trauma cases, preferably at the trauma room or at primary revisions of open fractures in order to expedite conjoint planning of further operations. An outpatient 
clinic with orthopedics and plastic surgeons for patients admitted from other hospitals was set up as well as multidisciplinary postoperative follow up of patients with trauma, and osteomyelitis following trauma to the extremities. A contract was established to ensure equal contribution of respective operating facilities and to ensure efficient rescheduling of elective surgery when emergent full day cases were to be planned with short notice.

To investigate the effects of this collaboration, a retrospective review was conducted for all patients who had suffered severe extremity trauma and received either a free or a pedicled flap for coverage of soft tissue defects $0-4$ years before and after the established collaboration. The main hypotheses were that this collaboration would decrease the time to soft tissue coverage as well as the in-hospital stay.

\section{Material and methods}

All consecutive patients who had suffered extremity trauma and who received either a free or a pedicled flap for coverage of soft tissue defects, between April 2004 and April 2012, were included. A comparison was made between the management of patients 4 years before the formalized collaboration (21 April 2004 - 20 April 2008) and 4 years after the formalized collaboration (21 April 2008 - 20 April 2012). Only patients with acute extremity trauma with exposed bone/open fracture or patients suffering from skin necrosis/infection after fracture surgery or chronic osteomyelitis following acute extremity trauma were selected for inclusion. Patients reconstructed after orthopedic tumor surgery or arthroplasty failures were not included. The included patients were identified by using the hospitals electronic operation planning system; Orbit (SYSteam Critical Care AB, Stockholm, Sweden). Patients were searched for using surgical procedure codes for different types of free and pedicled flaps. Time to flap coverage was counted from trauma/ admission at Karolinska University Hospital, or in the secondary cases from occurrence of wounds, fistulation or exposed bone. In case of a failed flap with demand for a secondary flap, time to flap coverage was counted to the first flap.

Open fractures were classified according to GustiloAnderson [9]. Fractures were classified strictly after the status at admission. Fractures classified as GIIIA, which in secondary revisions developed into IIIB due to tissue necrosis or infection were not reclassified.

In order to investigate the impact of the collaboration on time to flap surgery after admission to the hospital, and the impact on the postoperative treatment and inhospital stay, only patients with acute extremity trauma including open fractures or soft tissue defects with exposed bone were selected for a sub analysis (Tables 1 and 2). Patients with skin necrosis/infection after fracture surgery, chronic osteomyelitis, or patients referred to Karolinska University Hospital later than 14 days after the trauma were excluded from sub analysis.

Operations/revisions included all surgery performed in an operating room on the patient due to the trauma and included; primary wound debridement, primary fixation with external fixation device, secondary fixation in those cases where it did not concur with flap surgery, wound revisions/Topical negative pressure (TNP) therapy-change, reoperations due to flap failure, additional split skin grafting, extraction of external or internal fixation, bone grafting, conversion to circular external fixation (Taylor spatial frame $\left.^{\mathrm{m}}\right)$, corrections due to angulation/malrotation of fractures. TNP was only used as a dressing and not as a method to close the wound.

The total in-hospital stay was calculated by adding all admissions for operations/revisions before and after flap coverage.

The time to complete soft tissue coverage after flap surgery was determined as no remaining skin wounds at clinical evaluation at follow up and accepted as stated in the respective patient charts. Fracture healing was determined by radiological and clinical evaluation retrieved from patient charts.

The project was done in accordance with a protocol approved by the Ethical Committee at Karolinska Institutet, Stockholm, Sweden. (Project 2013/307-31/2).

The obtained results were statistically compared using a Mann-Whitney test in GraphPad Prism 5.0 (GraphPad Software Inc., CA). A p-value of $\leq 0.05$ was considered statistically significant. Results were presented with median values.

\section{Results}

In total, 52 patients ( 36 males, 16 females) treated with a free or pedicled flap were identified during the 8 year study period. One patient was included in both groups as she received one flap before and one after the start of the collaboration (53 cases). In total these patients received 57 flaps. 13 flaps were performed in the 4 years before the start of the collaboration, of which 5 were free flaps and 8 were pedicled flaps. 44 flaps were performed in the 4 years after the start of the collaboration, of which 21 were free flaps and 23 were pedicled flaps. Patients and choice of flaps is stated in Tables 1 and 2. All 5 free flaps performed before the collaboration survived. Total flap necrosis occurred in 2 out of 21 free flaps after the start of the collaboration. No partial necrosis was seen in free flaps. Total flap necrosis was seen in 1 pedicled flap before the start of the collaboration and 2 after the start of the collaboration. Partial flap necrosis was seen in 6 of the pedicled flaps ( 1 before and 5 after the start of the collaboration). The median age at the time of flap reconstruction before the start of the collaboration was 45 (21-64) 
Table 1 Patients treated before start of multidisciplinary collaboration

\begin{tabular}{|c|c|c|c|c|c|c|c|c|}
\hline & Sex & Age (y) & Case & Trauma & Gustilo & Flap & Time to flap (d) & Time to coverage (d) \\
\hline \multicolumn{9}{|c|}{$\begin{array}{l}\text { Free flaps } \\
\text { included }\end{array}$} \\
\hline 1 & M & 59 & Distal tibia fracture & $\begin{array}{l}\text { Excavator } \\
\text { accident }\end{array}$ & GIIIB & $\begin{array}{l}\text { Latissimus dorsi } \\
\text { muscle }\end{array}$ & 6 & 129 \\
\hline 2 & M & 42 & $\begin{array}{l}\text { Proximal tibia fracture, } \\
\text { fibula fracture, femoral } \\
\text { fracture, humeral fracture }\end{array}$ & MC accident & GIIIB & $\begin{array}{l}\text { Fibula osteocutaneous } \\
\text { flap (Medial gastro- } \\
\text { cnemius flap) }\end{array}$ & $49(0)$ & $\begin{array}{l}425 \text { Failed Medial } \\
\text { gastrocnemius flap }\end{array}$ \\
\hline 3 & M & 48 & Distal femoral fracture & Bus accident & GIIIC & $\begin{array}{l}\text { Latissimus dorsi } \\
\text { muscle }\end{array}$ & 7 & $\begin{array}{l}\text { Amputation } 2 \mathrm{~d} \\
\text { after flap }\end{array}$ \\
\hline \multicolumn{9}{|c|}{$\begin{array}{l}\text { Free flaps } \\
\text { excluded }\end{array}$} \\
\hline 4 & M & 54 & $\begin{array}{l}\text { Osteomyelitis calcaneal } \\
\text { fracture } 1 \text { year earlier }\end{array}$ & MC accident & GIIIA & Gracilis muscle & 360 & 7 \\
\hline 5 & M & 21 & $\begin{array}{l}\text { Osteomyelitis proximal tibia } \\
\text { after fracture } 5 \text { years earlier }\end{array}$ & $\begin{array}{l}\text { Moped } \\
\text { accident }\end{array}$ & GIIIB & $\begin{array}{l}\text { Latissimus dorsi } \\
\text { muscle }\end{array}$ & 5 years & 65 \\
\hline \multicolumn{9}{|c|}{$\begin{array}{l}\text { Pedicled flap } \\
\text { included }\end{array}$} \\
\hline 1 & M & 26 & $\begin{array}{l}\text { Multitrauma, calcaneal } \\
\text { fractures with skin necrosis }\end{array}$ & Fall injury & GIIIA & Sural island flap & 220 & 21 \\
\hline 2 & M & 60 & Diaphyseal tibia fracture & Fall injury & GIIIB & $\begin{array}{l}\text { Fasciocutaneous } \\
\text { rotation flap }\end{array}$ & 26 & 52 \\
\hline \multicolumn{9}{|c|}{$\begin{array}{l}\text { Pedicled flap } \\
\text { excluded }\end{array}$} \\
\hline 3 & M & 64 & $\begin{array}{l}\text { Tibia condyle fracture and } \\
\text { abundant soft tissue injury }\end{array}$ & $\begin{array}{l}\text { Outboard } \\
\text { motor } \\
\text { accident }\end{array}$ & GIIIC & $\begin{array}{l}\text { Lateral gastrocnemius } \\
\text { muscle }\end{array}$ & 26 & 72 \\
\hline 4 & $\mathrm{~F}$ & 30 & $\begin{array}{l}\text { Tibia pilon fracture, } \\
\text { secondary skin necrosis } \\
\text { after surgery }\end{array}$ & Fall injury & - & Sural island flap & 38 & 4 \\
\hline 5 & $\mathrm{~F}$ & 54 & $\begin{array}{l}\text { Osteomyelitis, distal tibia } \\
\text { fracture } 2 \text { years earlier, skin } \\
\text { necrosis after secondary } \\
\text { surgery }\end{array}$ & Fall injury & - & $\begin{array}{l}\text { Fasciocutaneous } \\
\text { transposition flap }\end{array}$ & 21 & $\begin{array}{l}\text { Partial flap necrosis } \\
\text { Free flap } 2011 \text { Pat } \\
15 \text { Table } 2\end{array}$ \\
\hline 6 & $\mathrm{~F}$ & 27 & $\begin{array}{l}\text { Multitrauma, proximal tibia } \\
\text { fracture, admitted } 1 \text { month } \\
\text { after trauma }\end{array}$ & Car accident & GIIIB & $\begin{array}{l}\text { Medial gastrocnemius } \\
\text { muscle }\end{array}$ & 51 & 57 \\
\hline 7 & M & 26 & $\begin{array}{l}\text { Diaphyseal tibia fracture, } \\
\text { admitted } 1.5 \text { months after } \\
\text { trauma }\end{array}$ & $\begin{array}{l}\text { Climbing } \\
\text { accident }\end{array}$ & GIIIB & Soleus muscle & 51 & 334 \\
\hline
\end{tabular}

Patients treated before start of multidisciplinary collaboration with a free or pedicled flap for soft tissue reconstruction after lower extremity trauma. Patients excluded in the subgroup analysis of acute extremity trauma were patients recieving flaps due to skin necrosis/infection after fracture surgery, chronic osteomyelitis, or patients referred to Karolinska University Hospital later than 14 days after the trauma. Time to flap is counted from trauma/admission at Karolinska University Hospital, or in the secondary cases from occurrence of wounds, fistulation or exposed bone. Time to complete soft tissue coverage after flap surgery was determined as no remaining skin wounds at clinical evaluation at follow up.

years. The median age after start of the collaboration was $47(15-86)$ years.

In 6 out of 12 cases before the start of the collaboration and 25 out of 42 cases after the start of the collaboration, flap treatment was due to acute extremity trauma with soft tissue defects. The rest were skin necrosis/infection after fracture surgery, chronic osteomyelitis, or patients referred to Karolinska University Hospital later than 14 days after the trauma. These patients were excluded from the analysis of treatment of acute extremity trauma to achieve a more uniform cohort for comparison.
Most fractures were graded as GIIIB, but there were also severe soft tissue injuries with exposed bone without fractures. The median time to flap coverage of acute extremity trauma was $6(0-51)$ days after the start of the collaboration, compared to $16.5(0-220)$ days before the start of the collaboration $(\mathrm{p}=0.283$ ) (Figure 1$)$. The median number of revisions/operations prior to flap coverage of acute extremity trauma was $3(0-7)$ after the start of the collaboration compared to $2.5(0-9)$ before the start of the collaboration $(\mathrm{p}=0.963)$ (Figure 2). The median number of revisions/operations following flap coverage of acute 
Table 2 Patients treated after start of multidisciplinary collaboration

\begin{tabular}{|c|c|c|c|c|c|c|c|c|}
\hline & Sex & Age (y) & Case & Trauma & Gustilo & Flap & Time to flap (d) & Time to coverage (d) \\
\hline \multicolumn{9}{|c|}{$\begin{array}{l}\text { Free flap } \\
\text { included }\end{array}$} \\
\hline 1 & M & 31 & Traumatic arm amputation & Work accident & GIIIC & Palmar free flap & 0 & 75 \\
\hline 2 & $\mathrm{~F}$ & 33 & $\begin{array}{l}\text { Calcaneus fracture, ankle } \\
\text { fracture, pelvic fracture }\end{array}$ & $\begin{array}{l}\text { Run over by a } \\
\text { lorry }\end{array}$ & GIIIB & $\begin{array}{l}\text { Latissimus dorsi } \\
\text { muscle }\end{array}$ & 4 & 203 \\
\hline 3 & M & 55 & $\begin{array}{l}\text { Diaphyseal tibia fracture } \\
\text { with bone defect }\end{array}$ & Gunshot & GIIIC & $\begin{array}{l}\text { Fibula osteo- } \\
\text { cutaneous flap }\end{array}$ & 4 & $\begin{array}{l}100 \text { Flap failure } \\
\text { and amputation }\end{array}$ \\
\hline 4 & M & 42 & Diaphyseal tibia fracture & Car accident & GIIIC & $\begin{array}{l}\text { Anterolateral } \\
\text { thigh flap }\end{array}$ & 11 & 192 \\
\hline 5 & M & 33 & $\begin{array}{l}\text { Multitrauma, diaphyseal } \\
\text { tibia fracture }\end{array}$ & MC accident & GIIIC & $\begin{array}{l}\text { Latissimus dorsi } \\
\text { muscle }\end{array}$ & 8 & 63 \\
\hline 6 & M & 61 & Distal tibia fracture & MC accident & GIIIC & $\begin{array}{l}\text { Latissimus dorsi } \\
\text { muscle }\end{array}$ & 3 & 87 \\
\hline 7 & $\mathrm{~F}$ & 46 & Distal tibia/fibula fracture & Riding accident & Gll & $\begin{array}{l}\text { Anterolateral } \\
\text { thigh flap }\end{array}$ & 7 & 31 \\
\hline 8 & $\mathrm{~F}$ & 31 & $\begin{array}{l}\text { Multitrauma, proximal } \\
\text { tibia/fibula fracture }\end{array}$ & Bicycle accident & GIIIC & $\begin{array}{l}\text { Latissimus dorsi } \\
\text { muscle }\end{array}$ & 16 & 148 \\
\hline 9 & M & 20 & $\begin{array}{l}\text { Multitrauma, distal tibia } \\
\text { fracture }\end{array}$ & Car accident & GIIIB & $\begin{array}{l}\text { Latissimus dorsi } \\
\text { muscle }\end{array}$ & 6 & $\begin{array}{l}23 \text { Amputation } 1 \text { year } \\
\text { after flap due to pain }\end{array}$ \\
\hline 10 & M & 27 & Soft tissue defect tibia & MC accident & - & Gracilis muscle & 7 & 104 \\
\hline 11 & M & 34 & Distal tibia fracture & MC accident & GIIIA & Gracilis muscle & 29 & 35 \\
\hline 12 & M & 66 & Ankle fracture & Work accident & GIIIA & $\begin{array}{l}\text { Anterolateral } \\
\text { thigh flap }\end{array}$ & 22 & 16 \\
\hline 13 & M & 36 & Distal tibia fracture & Car accident & GIIIA & Gracilis muscle & 3 & 21 \\
\hline 14 & M & 27 & $\begin{array}{l}\text { Multitrauma, tibia fracture } \\
\text { with bone defect }\end{array}$ & MC accident & GIIIC & $\begin{array}{l}\text { Fibula osteo- } \\
\text { cutaneous flap }\end{array}$ & 5 & 10 \\
\hline \multicolumn{9}{|c|}{$\begin{array}{l}\text { Free flap } \\
\text { excluded }\end{array}$} \\
\hline 15 & $\mathrm{~F}$ & 60 & $\begin{array}{l}\text { Osteomyelitis, distal tibia } \\
\text { fracture } 6 \text { years earlier }\end{array}$ & Fall injury & - & $\begin{array}{l}\text { Anterolateral } \\
\text { thigh flap }\end{array}$ & 5.5 years & $\begin{array}{l}40 \text { Pedicled flap } \\
2006 \text { Pat } 5 \text { Table } 1\end{array}$ \\
\hline 16 & M & 32 & $\begin{array}{l}\text { Infection after distal tibia } \\
\text { fracture, referred } 2 \text { months } \\
\text { after trauma }\end{array}$ & Car accident & GIIIB & $\begin{array}{l}\text { Anterolateral thigh } \\
\text { flap (Sural island flap) }\end{array}$ & $120(105)$ & $\begin{array}{l}42 \text { Failed Sural } \\
\text { island flap }\end{array}$ \\
\hline 17 & $\mathrm{~F}$ & 53 & $\begin{array}{l}\text { Distal tibia fracture, } \\
\text { secondary skin necrosis after } \\
\text { surgery }\end{array}$ & Fall injury & - & $\begin{array}{l}\text { Anterolateral } \\
\text { thigh flap }\end{array}$ & 456 & 29 \\
\hline 18 & M & 76 & $\begin{array}{l}\text { Trimalleolar ankle fracture, } \\
\text { referred } 2.5 \text { months after } \\
\text { trauma }\end{array}$ & Fall injury & GIIIA & Gracilis muscle & 105 & 128 \\
\hline 19 & M & 44 & $\begin{array}{l}\text { Osteomyelitis, tibia fracture } \\
15 \text { months earlier }\end{array}$ & Moped accident & GIIIC & $\begin{array}{l}\text { Latissimus dorsi } \\
\text { muscle }\end{array}$ & 432 & 24 \\
\hline 20 & $\mathrm{~F}$ & 63 & $\begin{array}{l}\text { Osteomyelitis, tibia pilon } \\
\text { fracture } 11 \text { months earlier }\end{array}$ & Fall injury & - & $\begin{array}{l}\text { Anterolateral } \\
\text { thigh flap } \\
\text { (Gracilis muscle) }\end{array}$ & $295(288)$ & $\begin{array}{l}134 \text { Failed gracilis } \\
\text { muscle }\end{array}$ \\
\hline \multicolumn{9}{|c|}{$\begin{array}{l}\text { Pedicled flap } \\
\text { included }\end{array}$} \\
\hline 1 & M & 40 & Proximal tibia fracture & MC accident & GIIIB & $\begin{array}{l}\text { Propeller flap } \\
\text { and lateral } \\
\text { gastrocnemius } \\
\text { muscle }\end{array}$ & 3 & $\begin{array}{l}457 \text { Partial flap } \\
\text { necrosis }\end{array}$ \\
\hline 2 & M & 70 & $\begin{array}{l}\text { Soft tissue injury to elbow, } \\
\text { and humeral fracture }\end{array}$ & $\begin{array}{l}\text { Lawn mower } \\
\text { accident }\end{array}$ & GIIIB & Radial forearm flap & 7 & 23 \\
\hline 3 & M & 17 & Soft tissue defect over knee & Moped accident & - & $\begin{array}{l}\text { Medial gastrocnemius } \\
\text { muscle }\end{array}$ & 11 & 9 \\
\hline
\end{tabular}


Table 2 Patients treated after start of multidisciplinary collaboration (Continued)

\begin{tabular}{|c|c|c|c|c|c|c|c|c|}
\hline 4 & M & 37 & Proximal tibia fracture & Bus accident & GIIIA & $\begin{array}{l}\text { Medial } \\
\text { gastrocnemius } \\
\text { muscle }\end{array}$ & 51 & 28 \\
\hline 5 & M & 31 & $\begin{array}{l}\text { Multitrauma, diaphyseal } \\
\text { tibia fracture }\end{array}$ & Car accident & GIIIB & $\begin{array}{l}\text { Fasciocutaneous } \\
\text { rotation flap }\end{array}$ & 4 & 16 \\
\hline 6 & M & 65 & Humeral fracture & Gunshot & GIIIB & $\begin{array}{l}\text { Latissimus dorsi } \\
\text { muscle }\end{array}$ & 3 & 35 \\
\hline 7 & $\mathrm{~F}$ & 64 & Proximal tibia fracture & $\begin{array}{l}\text { Run over by } \\
\text { a bus }\end{array}$ & GIIIA & $\begin{array}{l}\text { Medial } \\
\text { gastrocnemius } \\
\text { muscle }\end{array}$ & 16 & 65 \\
\hline 8 & $\mathrm{~F}$ & 15 & Proximal tibia fracture & Moped accident & GIIIB & $\begin{array}{l}\text { Medial } \\
\text { gastrocnemius } \\
\text { muscle }\end{array}$ & 1 & 21 \\
\hline 9 & $\mathrm{~F}$ & 43 & $\begin{array}{l}\text { Multitrauma, soft } \\
\text { tissue defect over } \\
\text { patella }\end{array}$ & Train accident & - & $\begin{array}{l}\text { Medial } \\
\text { gastrocnemius } \\
\text { muscle }\end{array}$ & 34 & 10 \\
\hline 10 & M & 55 & Diaphyseal tibia fracture & Bicycle accident & GIIIB & Soleus muscle & 4 & 4 \\
\hline 11 & M & 36 & Radial fractures & Gunshot & GIIIC & $\begin{array}{l}\text { Fasciocutaneous } \\
\text { transposition flap }\end{array}$ & 0 & $\begin{array}{l}15 \text { Partial flap } \\
\text { necrosis }\end{array}$ \\
\hline \multicolumn{9}{|c|}{$\begin{array}{l}\text { Pedicled flap } \\
\text { excluded }\end{array}$} \\
\hline 12 & $\mathrm{~F}$ & 59 & $\begin{array}{l}\text { Osteomyelitis, ankle fracture } \\
5.5 \text { years earlier }\end{array}$ & Fall injury & - & $\begin{array}{l}\text { Extensor digitorum } \\
\text { brevis muscle }\end{array}$ & 5.5 years & 112 \\
\hline 13 & M & 55 & $\begin{array}{l}\text { Distal tibia fracture } 7 \text { years } \\
\text { earlier, secondary skin } \\
\text { necrosis after surgery }\end{array}$ & Fall injury & - & $\begin{array}{l}\text { Extensor digitorum } \\
\text { brevis muscle }\end{array}$ & 7 years & 49 \\
\hline 14 & M & 47 & $\begin{array}{l}\text { Tibia pilon fracture, } \\
\text { secondary skin necrosis after } \\
\text { surgery }\end{array}$ & Fall injury & - & Sural island flap & 28 & $\begin{array}{l}143 \text { Partial flap } \\
\text { necrosis }\end{array}$ \\
\hline 15 & M & 55 & $\begin{array}{l}\text { Osteomyelitis, tibia condyle } \\
\text { fracture } 17 \text { months earlier } \\
\text { secondary skin necrosis after } \\
\text { surgery }\end{array}$ & MC accident & - & $\begin{array}{l}\text { Medial } \\
\text { gastrocnemius } \\
\text { muscle }\end{array}$ & 156 & $\begin{array}{l}8 \text { Amputation } 8 \mathrm{~d} \\
\text { after flap }\end{array}$ \\
\hline 16 & $\mathrm{~F}$ & 71 & $\begin{array}{l}\text { Ulnar fracture, secondary } \\
\text { skin necrosis after surgery }\end{array}$ & Fall injury & - & $\begin{array}{l}\text { Fasciocutaneous } \\
\text { rotation flap }\end{array}$ & 63 & 18 \\
\hline 17 & $\mathrm{~F}$ & 86 & $\begin{array}{l}\text { Proximal tibia fracture, } \\
\text { secondary skin necrosis after } \\
\text { surgery }\end{array}$ & Fall injury & - & $\begin{array}{l}\text { Medial } \\
\text { gastrocnemius } \\
\text { muscle }\end{array}$ & 61 & 11 \\
\hline 18 & $\mathrm{~F}$ & 62 & $\begin{array}{l}\text { Distal tibia fracture } 3 \text { years } \\
\text { earlier, wound infection after } \\
\text { hardware removal }\end{array}$ & Fall injury & - & Soleus muscle & 239 & $\begin{array}{l}17 \text { Partial flap } \\
\text { necrosis }\end{array}$ \\
\hline 19 & M & 63 & $\begin{array}{l}\text { Multitrauma, patellar } \\
\text { fracture, secondary skin } \\
\text { necrosis after surgery }\end{array}$ & Car accident & - & $\begin{array}{l}\text { Medial } \\
\text { gastrochnemius } \\
\text { muscle } \\
\text { (Fasciocutaneous } \\
\text { rotation flap) }\end{array}$ & (103) 51 & $\begin{array}{l}190 \text { Partial flap } \\
\text { necrosis rotation } \\
\text { flap }\end{array}$ \\
\hline 20 & $\mathrm{~F}$ & 67 & $\begin{array}{l}\text { Distal tibia fracture, } \\
\text { secondary skin necrosis after } \\
\text { surgery }\end{array}$ & Fall injury & - & Propeller flap & 135 & $\begin{array}{l}\text { Not healed by } \\
\text { inclusion }\end{array}$ \\
\hline 21 & M & 76 & $\begin{array}{l}\text { Distal tibia fracture, } \\
\text { secondary skin } \\
\text { necrosis after plaster }\end{array}$ & $\begin{array}{l}\text { Wheelchair } \\
\text { accident }\end{array}$ & - & Sural island flap & 42 & $\begin{array}{l}\text { Flap failure, } \\
\text { Amputation } 70 \mathrm{~d} \text {. } \\
\text { after flap }\end{array}$ \\
\hline
\end{tabular}

Patients treated after start of multidisciplinary collaboration with a free or pedicled flap for soft tissue reconstruction after lower extremity trauma. Patients excluded in the subgroup analysis of acute extremity trauma were patients recieving flaps due to skin necrosis/infection after fracture surgery, chronic osteomyelitis, or patients referred to Karolinska University Hospital later than 14 days after the trauma. Time to flap is counted from trauma/admission at Karolinska University Hospital, or in the secondary cases from occurrence of wounds, fistulation or exposed bone. Time to complete soft tissue coverage after flap surgery was determined as no remaining skin wounds at clinical evaluation at follow up. 


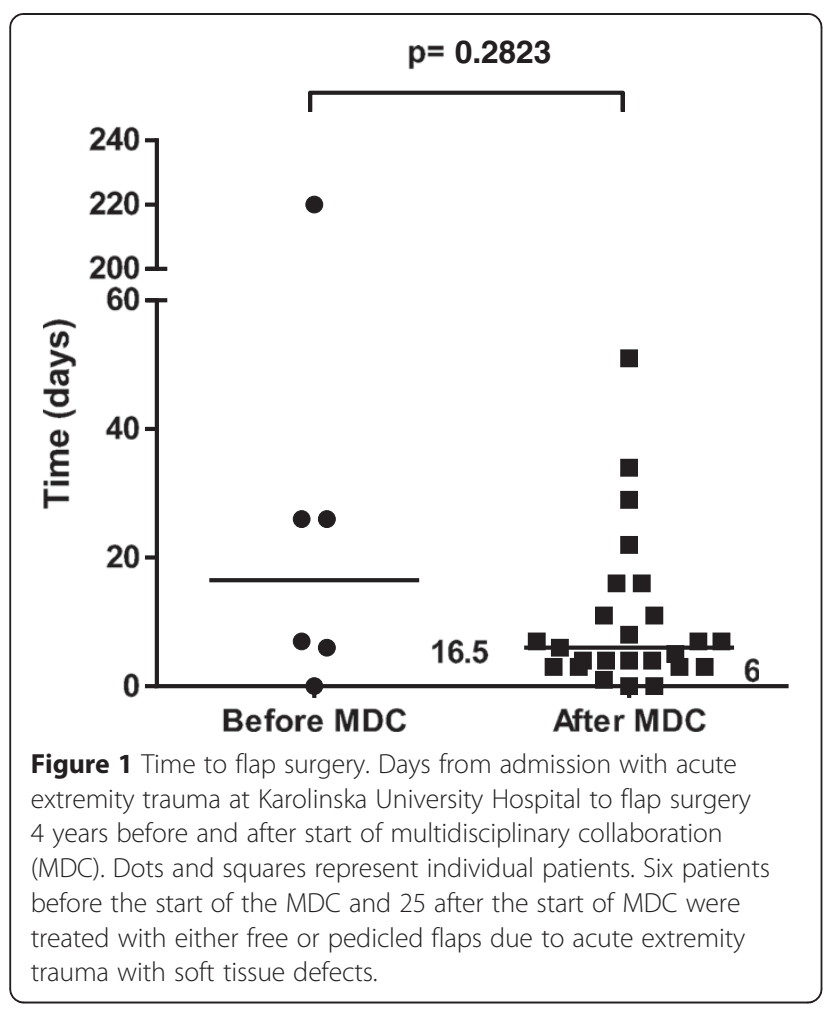

extremity trauma was $1(1-10)$ after the start of the collaboration compared to $4(0-10)$ before the start of the collaboration $(\mathrm{p}=0.046)$ (Figure 3).The median time for complete soft tissue coverage after flap surgery of acute extremity trauma was 33 (9-203) days after the start of the collaboration compared to $62(2-425)$ days before the

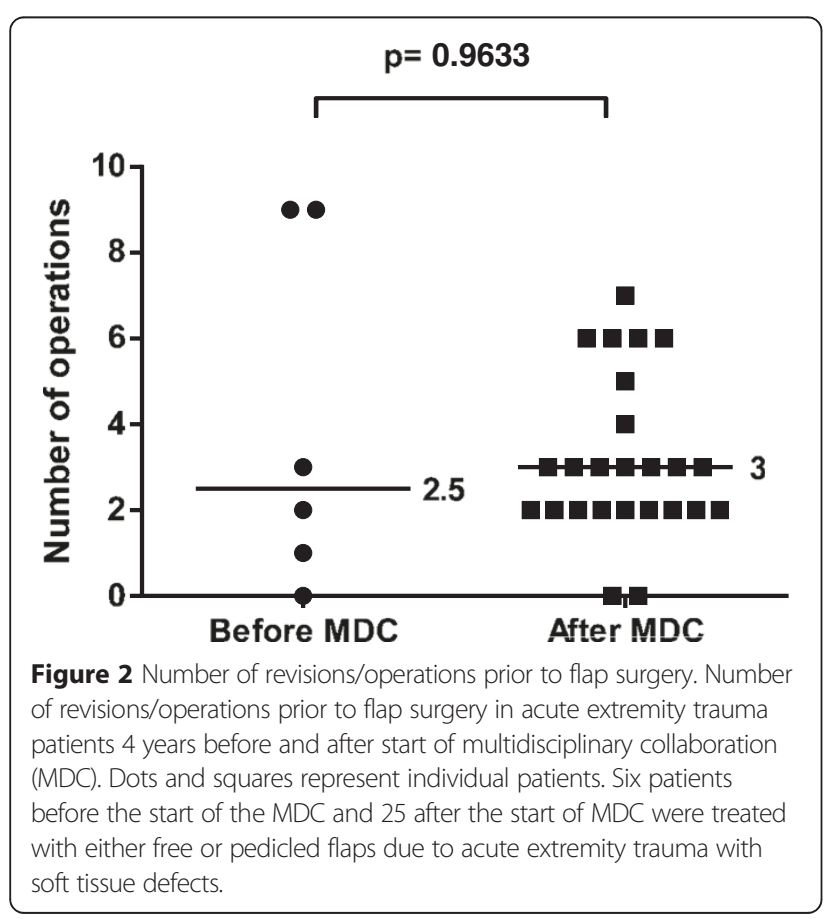

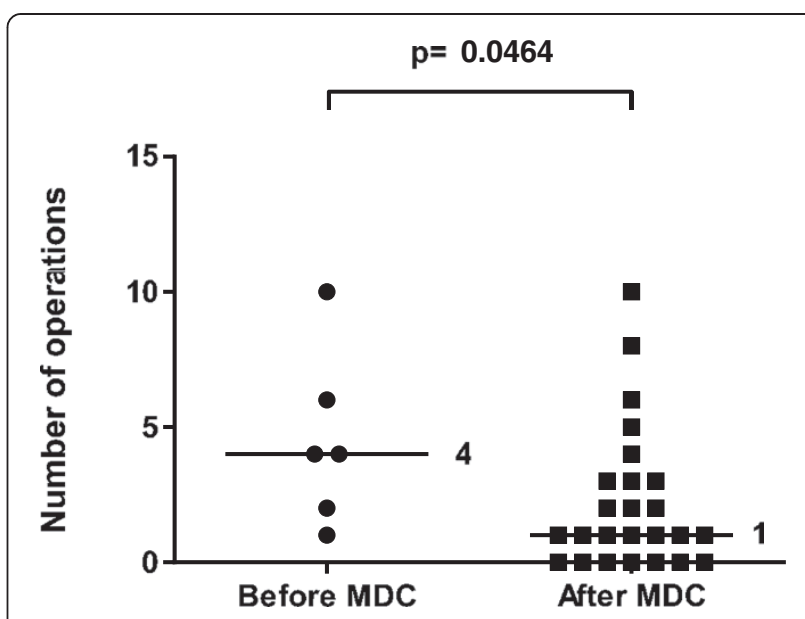

Figure 3 Number of revisions/operations after flap surgery. Number of revisions/operations after flap surgery in acute extremity trauma patients 4 years before and after start of multidisciplinary collaboration (MDC). Dots and squares represent individual patients. Six patients before the start of the MDC and 25 after the start of MDC were treated with either free or pedicled flaps due to acute extremity trauma with soft tissue defects.

start of the collaboration $(\mathrm{p}=0.518)$. The median time in hospital after acute extremity trauma was reduced from $67.5(16-88)$ days to $29(8-121)$ days after the start of the collaboration ( $\mathrm{p}=0.044)$ (Figure 4). All included fractures healed. Time to fracture healing was 296 days before the start of the collaboration and 256 days after the start of the collaboration $(p=0.594)$. Only 5 out of 6 patients before and 16 out of 25 patients after the start of the

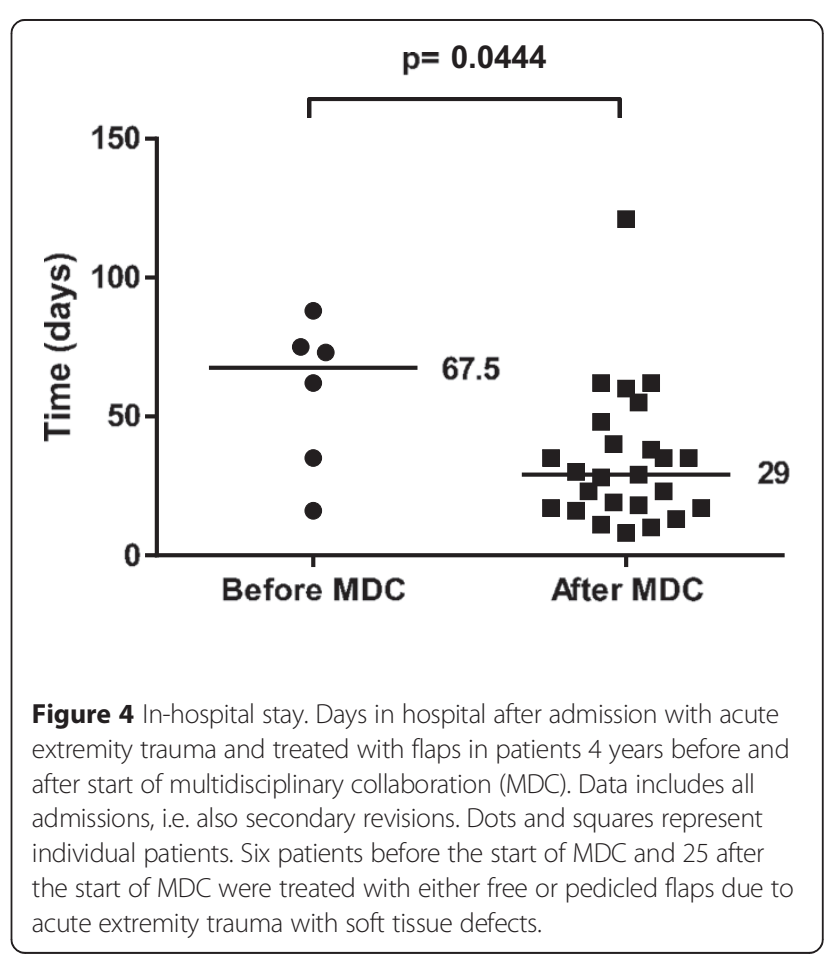


collaboration were possible to follow to fracture healing. One patient was amputated after flap treatment before the start of the collaboration. After the start of the collaboration, four patients had soft tissue defects with exposed bone without fractures, one had a primary traumatic amputation, two were amputated prior to flap treatment and one patient was not healed at the time of inclusion in the study. One of the patients healed, but was amputated one year after flap coverage due to pain.

\section{Discussion}

Severe extremity trauma with soft tissue defects is a difficult task for both the patient and health care provider. In order to optimize the outcome for the patients we have improved our care of the patients. There is a growing body of evidence that these complex extremity injuries are best handled by a multidisciplinary team of experienced plastic and orthopedic surgeons $[8,10]$. The present study clearly demonstrates an increase of flap reconstruction in acute trauma patients and patients with secondary wounds following acute extremity trauma after the start of a multidisciplinary collaboration. This is hardly explained by natural variation, as the observation time is 8 years, and it seems unlikely that variation could explain a 3.5-fold increase as in our series. In 2007 all high energy trauma in Stockholm County was regionalized to Karolinska University Hospital. This has resulted in a 0.75 -fold increase of trauma patients. Patients with severe extremity trauma is now primary taken to Karolinska University Hospital. Prior to 2007 the reference policy was less strict and hence not all cases were treated at Karolinska. The severity of orthopedic trauma has not changed during the years. Occasionally patients with open fractures are still treated in other hospitals in Stockholm, but they are usually referred to Karolinska after external fixation and primary revision. We have also seen an increase in both acute and secondary cases referred from other hospitals in the region as the collaboration has become known. The regionalization only cannot explain a 3.5-fold increase of flaps. We believe that the main reason is an increased awareness among our orthopedic surgeons to refer extremity trauma patients for plastic surgical consultation. The collaboration has also resulted in a more rapid communication, and quicker decisions.

The major limitation in this report is the small sample size. Since the patient group treated before start of the collaboration is small, the sub analysis of acute extremity trauma patients cannot provide accurate statistical comparison, and the reader should interpret the significance between groups with care. One of the acute trauma patients included before start of the MDC was not covered before 220 days after trauma. This patient had bilateral calcaneal fractures after a fall injury, classified as a
Gustilo IIIA due to crush wounds on the heels. During the following days he developed skin necrosis on the right heal. Due to a more conservative attitude towards small wounds in extremity trauma before the start of MDC, there was an attempt of secondary healing. Today, this patient would probably have received a free flap in the near time period of the trauma. To reduce the effects of this "outlier" statistically we have chosen to use a nonparametric method, and present median values. This patient was not an outlier in comparisons other than time to flap.

The median time to flap coverage after the start of the collaboration was 6 days, which still is not satisfactory. Institutional factors such as available OR time and support staff may make immediate reconstruction of extremity injuries impossible. There can also be other concomitant injury which first has to be dealt with, and the extent of soft tissue injury is sometimes difficult to determine early in the process. Whereas Godina [1] clearly states the superiority of reconstruction within 3 days, other authors have shown excellent results with late coverage [11-13]. There was no difference in outcome or flap failure in our series when comparing subgroups of patients; flap coverage $<3$ days vs. $>3$ days, or flap coverage $<6$ days vs. $>6$ days (data not shown). It is not possible to draw overreaching conclusions from this due to the relative small groups in comparison to the work by Godina [1]. One key to earlier flap coverage is to perform fewer revisions. After the start of the collaboration we still had a median number of 3 operations/revisions before flap coverage. Only one radical debridement performed by the most experienced surgeons before flap coverage has been set up as a future goal for the team.

The introduction of a collaboration had a significant impact on the in hospital stay. The median time in hospital after acute extremity trauma with soft tissue defects decreased from 68.5 days to 29 days. In the 25 patients after the start of the collaboration, this would correspond to 988 hospital days, and a total cost reduction of 1.170000 USD. In times where cost-analysis is crucial in public health care, this reduction which corresponds to $70 \%$ of one hospital bed/year is obviously beneficial. The reduction of hospital stay may be explained by the decrease in revisions/operations after flap coverage, which may be related to a shorter time to coverage.

The incidence of open tibia fractures in the Swedish population is $2.3 / 10^{5}$ /year [6], which corresponds to about 220 patients per year, of which 27\% [5] have a Gustilo Type III fracture. Because of the low and declining incidence, centralization of these difficult fractures has been suggested $[8,14]$. We believe that these fractures should be dealt with in a center with a close collaboration between orthopedic and plastic surgeons. The experience after the Haiti earthquake with an orthoplastic limb salvage team 
are in concordance, showing favorable amputation rate and a more efficient planning of surgical workload [10].

\section{Conclusion}

To conclude, the formalized collaboration has led to an increased number of flap coverage in extremity trauma patients. The acute cases have a shorter in-hospital stay, and fewer postoperative revisions after the start of the collaboration. However, if this has resulted in a decreased number of amputations and improved long-term outcome needs to be further analyzed in a larger cohort. Our collaboration has also led to awareness among our orthopedic colleagues to establish an early contact with a plastic surgeon when a patient has an open fracture with soft tissue damage. The goal is that the plastic surgeon should be contacted from the emergency room in order to participate at the first operative revision to be able to plan for further surgery. Routines are also created for coordination of operative resources, and postoperative flap surveillance. Finally, this formal collaboration gives us better possibilities for quality control and evaluation. Our experience leads us to recommend other centers to establish a similar set-up for early multidisciplinary treatment of this patient group.

\section{Consent}

Written informed consent was obtained from the patients for the publication of this report.

\section{Competing interests}

The authors declare that they have no competing interests.

\section{Authors' contributions}

PS: Surgery, collection of data and writing of manuscript. YG: Collection of data and writing of manuscript. MH: Surgery, review of patient data and writing of manuscript. ADS: Surgery and writing of manuscript. KL: Surgery, review of patient data and writing of manuscript. KÅJ: Surgery and writing of manuscript. All authors read and approved the final manuscript.

\section{Author details \\ 1 Department of Molecular Medicine and Surgery, Section of Plastic Surgery, Karolinska Institutet and Karolinska University Hospital Stockholm, Stockholm, Sweden. ${ }^{2}$ Department of Orthopedic Surgery Västerås Hospital and Department of Molecular Medicine and Surgery, Karolinska Institutet Stockholm, Stockholm, Sweden. ${ }^{3}$ Department of Molecular Medicine and Surgery, Section of Orthopedics and Sports Medicine, Karolinska Institutet and Karolinska University Hospital Stockholm, Stockholm, Sweden.}

Received: 29 May 2014 Accepted: 13 March 2015

Published online: 15 April 2015

\section{References}

1. Godina M. Early microsurgical reconstruction of complex trauma of the extremities. Plast Reconstr Surg. 1986;78:285-92.

2. Tielinen $L$, Lindahl JE, Tukiainen EJ. Acute unreamed intramedullary nailing and soft tissue reconstruction with muscle flaps for the treatment of severe open tibial shaft fractures. Injury. 2007;38:906-12.

3. Gopal S, Majumder S, Batchelor AG, Knight SL, De Boer P, Smith RM. Fix and flap: the radical orthopaedic and plastic treatment of severe open fractures of the tibia. J Bone Joint Surg. 2000;82:959-66.

4. Levin LS. Early versus delayed closure of open fractures. Injury. 2007:38:896-9.
5. Meling T, Harboe K, Soreide K. Incidence of traumatic long-bone fractures requiring in-hospital management: a prospective age- and gender-specific analysis of 4890 fractures. Injury. 2009;40:1212-9.

6. Weiss RJ, Montgomery SM, Ehlin A, Al Dabbagh Z, Stark A, Jansson KA. Decreasing incidence of tibial shaft fractures between 1998 and 2004: information based on 10,627 Swedish inpatients. Acta Orthop. 2008;79:526-33.

7. Heitmann C, Levin LS. The orthoplastic approach for management of the severely traumatized foot and ankle. J Trauma. 2003;54:379-90.

8. Naique SB, Pearse M, Nanchahal J. Management of severe open tibial fractures: the need for combined orthopaedic and plastic surgical treatment in specialist centres. J Bone Joint Surg. 2006;88:351-7.

9. Gustilo RB, Mendoza RM, Williams DN. Problems in the management of type III (severe) open fractures: a new classification of type III open fractures. J Trauma. 1984;24:742-6.

10. Clover AJ, Rannan-Eliya S, Saeed W, Buxton R, Majumder S, Hettiaratchy SP, et al. Experience of an orthoplastic limb salvage team after the Haiti earthquake: analysis of caseload and early outcomes. Plast Reconstr Surg. 2011;127:2373-80.

11. Karanas $Y L$, Nigriny J, Chang J. The timing of microsurgical reconstruction in lower extremity trauma. Microsurgery. 2008;28:632-4.

12. Hill JB, Vogel JE, Sexton KW, Guillamondegui OD, Corral GA, Shack RB. Re-evaluating the paradigm of early free flap coverage in lower extremity trauma. Microsurgery. 2013;33:9-13.

13. Francel TJ, Vander Kolk CA, Hoopes JE, Manson PN, Yaremchuk MJ. Microvascular soft-tissue transplantation for reconstruction of acute open tibial fractures: timing of coverage and long-term functional results. Plast Reconstr Surg. 1992:89:478-87. discussion 488-479.

14. Court-Brown CM, Rimmer S, Prakash U, McQueen MM. The epidemiology of open long bone fractures. Injury. 1998;29:529-34

\section{Submit your next manuscript to BioMed Central and take full advantage of:}

- Convenient online submission

- Thorough peer review

- No space constraints or color figure charges

- Immediate publication on acceptance

- Inclusion in PubMed, CAS, Scopus and Google Scholar

- Research which is freely available for redistribution 